International Mathematical Forum, Vol. 9, 2014, no. 35, 1707 - 1718 HIKARI Ltd, www.m-hikari.com http://dx.doi.org/10.12988/imf.2014.410176

\title{
Split Equality Fixed Point Problem for Strictly Pseudocontractive Mappings ${ }^{1}$
}

\author{
Zhaoli Ma ${ }^{2,4}$, Wen Duan ${ }^{3}$ and RuiJuan Liu ${ }^{2}$ \\ 2 School of Information Engineering \\ College of Arts and Sciences \\ Yunnan Normal University \\ Kunming, Yunnan, 650222, P.R. China \\ ${ }^{4}$ Corresponding author \\ 3 Yaoan Branch, Chuxiong State Co., Yunnan Tobacco Co. \\ Chuxiong, Yunnan, 675300, P.R. China
}

Copyright (c) 2014 Zhaoli Ma, Wen Duan and RuiJuan Liu. This is an open access article distributed under the Creative Commons Attribution License, which permits unrestricted use, distribution, and reproduction in any medium, provided the original work is properly cited.

\begin{abstract}
In this paper, we introduce a new algorithm for solving the split equality fixed point problem of strictly pseudocontractive mapping in the framework of infinite-dimensional real Hilbert spaces. The strong and weak convergence theorems are obtained. Our results presented in this paper improve and extend some recent corresponding results.
\end{abstract}

Mathematics Subject Classifications: 47H09, 47J25

Keywords: Split equality problem; Split feasibility problem; Strictly pseudocontractive mapping; bounded linear operator

\section{Introduction}

For modeling inverse problems which arise form phase retrievals and in medical

\footnotetext{
${ }^{1}$ This work was supported by the Scientific Research Foundation of the College of Arts and Sciences Yunnan Normal (Grant No. 13KJY0023).
} 
image reconstruction [1], in 1994 Censor and Elfving [2] first introduced the following split feasibility problem (SFP) in finite-dimensional Hilbert spaces:

Let $C$ and $Q$ be the nonempty closed convex subsets of the Hilbert spaces $H_{1}$ and $H_{2}$, respectively, $A: H_{1} \rightarrow H_{2}$ be a bounded linear operator. The split feasibility problem (SFP) is formulated as finding a point $x^{*}$ with the property

$$
x^{*} \in C \text { and } A x^{*} \in Q .
$$

The SFP has been found that it can be used in many areas such as image restoration, computer tomograph, and radiation therapy treatment planing [3-5]. Some methods have been proposed to solve split feasibility problem, see for instance $[1,6-9]$.

Assuming that the SFP is consistent (i.e.(1.1)) has a solution), it is not hard to see that

$$
x^{*}=P_{C}\left(I+\gamma A^{*}\left(P_{Q}-I\right) A x^{*}, \forall x \in C,\right.
$$

where $P_{C}$ and $P_{Q}$ are the (orthogonal) projection onto $C$ and $Q$, respectively, $\gamma>0$, and $A^{*}$ denotes the adjoint of $A$. That is, $x^{*}$ solves the $S F P(1.1)$ if and only if $x^{*}$ solves the fixed point equation (1.2) [see 10]. This implies that we can use fixed point algorithms to solve $S F P$.

Recently, Moudafi [11] introduced the following new split feasibility problems:

Let $H_{1}, H_{2}, H_{3}$ be real Hilbert spaces, $C \subset H_{1}, Q \subset H_{2}$ be two nonempty closed convex sets, $A: H_{1} \rightarrow H_{3}, B: H_{2} \rightarrow H_{3}$ be two bounded linear operators. The new split feasibility problem is to

$$
\text { find } x^{*} \in C, y^{*} \in Q \quad \text { such that } A x^{*}=B y^{*} \text {. }
$$

This allows asymmetric and partial relations between the variables $x$ and $y$.

It is easy to see that the problem (1.3) reduces to the problem (1.1) as $H_{2}=H_{3}$ and $B=I$ ( $I$ stands for the identity mapping from $H_{2}$ to $H_{2}$ ) in (1.3). Therefore the new split feasibility problem (1.3) proposed by Moudafi is generalization of the split feasibility problem (1.1).

Since each nonempty closed convex subset of a Hilbert space can be regards as a set of fixed points of a projection, in [12], Moudafi proposed a new split feasibility problem, which is also called split equality fixed point problem, that is,

$$
\text { Find } x \in C, y \in Q \text { such that } A x=B y, \text { with } C:=\text { FixU, } Q:=\text { FixT, }
$$

where $U: H_{1} \rightarrow H_{1}$ and $T: H_{2} \rightarrow H_{2}$ are two nonlinear operators with nonempty fixed point sets $C:=$ FixU, $Q:=$ FixT, $A: H_{1} \rightarrow H_{3}$ and $B: H_{2} \rightarrow H_{3}$ are two bounded linear operators. This allows asymmetric and partial relations between the variables $x$ and $y$. The interest is to cover many situations, for instance in 
decomposition methods for $P D E^{\prime} s$, applications in game theory and in intensitymodulated radiation therapy.

We use $\Omega$ to denote the set of solutions of the new split feasibility problem (1.4), i.e.,

$$
\Omega=\{(x, y): A x=B y, x \in C, y \in Q\} .
$$

To solve the (1.4), Modaufi [12] presented the following simultaneous iterative method and obtained weak convergence theorem:

$$
(S I M-F P P)\left\{\begin{array}{l}
x_{k+1}=U\left(x_{k}-\gamma_{k} A^{*}\left(A x_{k}-B y_{k}\right)\right) ; \\
y_{k+1}=T\left(y_{k}+\gamma_{k} B^{*}\left(A x_{k}-B y_{k}\right)\right),
\end{array}\right.
$$

where $H_{1}, H_{2}, H_{3}$ are real Hilbert spaces, $U: H_{1} \rightarrow H_{1}, T: H_{2} \rightarrow H_{2}$ are two firmly quasi-nonexpansive mappings, $A: H_{1} \rightarrow H_{3}, B: H_{2} \rightarrow H_{3}$ are two bounded linear operators, $A^{*}$ and $B^{*}$ are the adjoint of $A$ and $B$, respectively, $C$ is the set of fixed points of $U$ and $Q$ is the set of fixed points of $T$.

Motivated by the work of Moudafi [11, 12], in this paper, we construct the following iterative algorithm to solve the split equality fixed point problem of strictly pseudocontractive mappings in real Hilbert spaces:

$$
\left\{\begin{array}{l}
\forall x_{1} \in H_{1}, \quad \forall y_{1} \in H_{2} \\
x_{n+1}=\alpha_{n} x_{n}+\left(1-\alpha_{n}\right) T_{1}\left(x_{n}-\gamma_{n} A^{*}\left(A x_{n}-B y_{n}\right)\right) \\
y_{n+1}=\alpha_{n} y_{n}+\left(1-\alpha_{n}\right) T_{2}\left(y_{n}+\gamma_{n} B^{*}\left(A x_{n}-B y_{n}\right)\right), \quad \forall n \geq 1
\end{array}\right.
$$

and to obtain the strong and weak convergence theorems for strictly pseudocontractive mapping which is more general than firmly quasi-nonexpansive mapping and nonexpansive mapping. Our results extend and improve the corresponding results of Moudafi [11,12].

\section{Preliminaries}

We first recall some definitions and lemmas which will be needed in proving our main results.

Definition 2.1. Let $H$ be a Hilbert space.

(1) A single-value mapping $T: H \rightarrow H$ is said to be demi-closed at origin, if for any sequence $\left\{x_{n}\right\} \subset H$ with $x_{n} \rightarrow x^{*}$, and $\left\|(I-T) x_{n}\right\| \rightarrow 0$, then we have $x^{*}=T x^{*}$.

(2) A single-value mapping $T: H \rightarrow H$ is said to be semi-compact, if for any bounded sequence $\left\{x_{n}\right\} \subset H$ with $\left\|(I-T) x_{n}\right\| \rightarrow 0$, then there exists a subsequence $\left\{x_{n_{i}}\right\} \subset\left\{x_{n}\right\}$ such that $\left\{x_{n_{i}}\right\}$ converges strongly to a point $x^{*} \in$ $H$. 
Definition 2.2. Let $H$ be a real Hilbert space.

(1) A mapping $T: H \rightarrow H$ is said to be nonexpansive if

$$
\|T x-T y\| \leq\|x-y\|, \quad \forall x, y \in H
$$

(2) A mapping $T: H \rightarrow H$ is said to be quasi-nonexpansive if

$$
\|T x-q\| \leq\|x-q\|, \quad \forall(x, q) \in H \times F(T) .
$$

(3) A mapping $T: H \rightarrow H$ is said to be L-Lipschitzian if there exists a constant $L>0$ such that

$$
\|T x-T y\| \leq L\|x-y\|, \quad \forall x, y \in H
$$

(4) A mapping $T: H \rightarrow H$ is said to be $k$-strictly pseudocontractive if there exists $k \in[0,1)$, such that

$$
\|T x-T y\|^{2} \leq\|x-y\|^{2}+k\|(I-T) x-(I-T) y\|^{2}, \quad \text { for } x, y \in H .
$$

It is obvious that $T$ is nonexpansive if and only if $T$ is a 0 -strict pseudocontraction. So, the class of strictly pseudocontractive mappings strictly includes the class of nonexpansive mappings.

Lemma 2.3.[13] Let $C$ be a nonempty closed convex subset of $H$ and let $T: C \rightarrow C$ be a k-strictly pseudocontractive mapping, then the following results hold:

(1) equation (2.4) is equivalent to

$$
<T x-T y, x-y>\leq\|x-y\|^{2}-\frac{1-k}{2}\|x-y-(T x-T y)\|^{2}, \quad \forall x, y \in C .
$$

(2) $T$ is Lipschitz continuous with a constant $\frac{1+k}{1-k}$, i.e.,

$$
\|T x-T y\| \leq \frac{1+k}{1-k}\|x-y\|, \quad \forall x, y \in C .
$$

(3) (Demi-closed principle) $I-T$ is demi-closed on $C$, that is,

$$
\text { if } x_{n} \rightarrow x^{*} \in C \text { and }(I-T) x_{n} \rightarrow 0 \text {, then } x^{*}=T x^{*} \text {. }
$$


It is clear that equation (2.4) is equivalent to

$$
<(I-T) x-(I-T) y, x-y>\geq \frac{1-k}{2}\|(I-T) x-(I-T) y\|^{2}, \quad \forall x, y \in C .
$$

Lemma 2.4. [12] Let $H$ be a Hilbert space and $\left\{\mu_{n}\right\}$ be a sequence in $H$ such that there exists a nonempty set $W \subset H$ satisfying:

(i) For every $\mu^{*} \in W, \lim _{n \rightarrow \infty}\left\|\mu_{n}-\mu^{*}\right\|$ exists.

(ii) Any weak-cluster point of the sequence $\left\{\mu_{n}\right\}$ belongs to $W$.

Then, there exists $\mu^{*} \in W$ such that $\left\{\mu_{n}\right\}$ weakly converges to $\mu^{*}$.

Lemma 2.5 ([14]) Let $H$ be a real Hilbert space, then for all $x, y \in H$, we have

$$
\|x-y\|^{2} \leq\|x\|^{2}-\|y\|^{2}-2<x-y, y>.
$$

\section{Main result}

Theorem 3.1. Let $H_{1}, H_{2}, H_{3}$ be real Hilbert spaces, let $T_{1}: H_{1} \rightarrow H_{1}$, $T_{2}: H_{2} \rightarrow H_{2}$ be two strictly pseudocontractive mappings for some $k \in[0,1)$, and $A: H_{1} \rightarrow H_{3}, B: H_{2} \rightarrow H_{3}$ be two bounded linear operators. Assume that the iteration scheme $\left\{\left(x_{n}, y_{n}\right)\right\}$ is defined as follows:

$$
\left\{\begin{array}{l}
\forall x_{1} \in H_{1}, \quad \forall y_{1} \in H_{2} \\
x_{n+1}=\alpha_{n} x_{n}+\left(1-\alpha_{n}\right) T_{1}\left(x_{n}-\gamma_{n} A^{*}\left(A x_{n}-B y_{n}\right)\right) ; \\
y_{n+1}=\alpha_{n} y_{n}+\left(1-\alpha_{n}\right) T_{2}\left(y_{n}+\gamma_{n} B^{*}\left(A x_{n}-B y_{n}\right)\right), \quad \forall n \geq 1 .
\end{array}\right.
$$

where $\lambda_{A}$ and $\lambda_{B}$ stand for the spectral radius of $A^{*} A$ and $B^{*} B$ respectively, $\left\{\alpha_{n}\right\}$ is a sequence in $(0,1)$ with $\frac{k+1}{2}<\alpha \leq \alpha_{n} \leq \beta<1$ (for some $\alpha, \beta \in(0,1)$ ), and $\left\{\gamma_{n}\right\}$ is a positive real sequence such that $\gamma_{n} \in\left(\varepsilon, \frac{2(1-\beta)}{\left((1-\alpha)^{2}+\beta^{2}\right)\left(\lambda_{A}+\lambda_{B}\right)}-\varepsilon\right.$ ) (for $\varepsilon$ small enough), $C:=F\left(T_{1}\right)$ and $Q:=F\left(T_{2}\right)$. If $\Omega \neq \emptyset$, then

(I) The sequence $\left\{\left(x_{n}, y_{n}\right)\right\}$ converges weakly to a solution $(x, y)$ of the problem (1.4).

(II) In addition, if $T_{1}, T_{2}$ are also semi-compact, then $\left\{\left(x_{n}, y_{n}\right)\right\}$ converges strongly to a solution of the problem (1.4).

\section{Proof:}

Now we prove the conclusion (I). 
Let $(x, y) \in \Omega$. Since $\|\cdot\|^{2}$ is convex and $T_{1}, T_{2}$ are strictly pseudocontractive mappings, we have

$$
\begin{aligned}
\left\|x_{n+1}-x\right\|^{2}= & \left\|\alpha_{n} x_{n}+\left(1-\alpha_{n}\right) T_{1}\left(x_{n}-\gamma_{n} A^{*}\left(A x_{n}-B y_{n}\right)\right)-x\right\|^{2} \\
= & \alpha_{n}^{2}\left\|x_{n}-x\right\|^{2}+\left(1-\alpha_{n}\right)^{2}\left\|T_{1}\left(x_{n}-\gamma_{n} A^{*}\left(A x_{n}-B y_{n}\right)\right)-x\right\|^{2} \\
& +2 \alpha_{n}\left(1-\alpha_{n}\right)<x_{n}-x, T_{1}\left(x_{n}-\gamma_{n} A^{*}\left(A x_{n}-B y_{n}\right)\right)-x> \\
\leq & \alpha_{n}^{2}\left\|x_{n}-x\right\|^{2}+\left(1-\alpha_{n}\right)^{2}\left\|x_{n}-\gamma_{n} A^{*}\left(A x_{n}-B y_{n}\right)-x\right\|^{2} \\
& +\left(1-\alpha_{n}\right)^{2} k\left\|\left(I-T_{1}\right)\left(x_{n}-\gamma_{n} A^{*}\left(A x_{n}-B y_{n}\right)\right)\right\|^{2} \\
& +2 \alpha_{n}\left(1-\alpha_{n}\right)<x_{n}-x, T_{1}\left(x_{n}-\gamma_{n} A^{*}\left(A x_{n}-B y_{n}\right)\right)-x>.
\end{aligned}
$$

By (2.8), we have

$$
\begin{aligned}
<x_{n}-x, T_{1} & \left(x_{n}-\gamma_{n} A^{*}\left(A x_{n}-B y_{n}\right)\right)-x> \\
= & <x_{n}-x, T_{1}\left(x_{n}-\gamma_{n} A^{*}\left(A x_{n}-B y_{n}\right)\right)-\left(x_{n}-\gamma_{n} A^{*}\left(A x_{n}-B y_{n}\right)\right) \\
& +x_{n}-\gamma_{n} A^{*}\left(A x_{n}-B y_{n}\right)-x> \\
= & <x_{n}-x,\left(T_{1}-I\right)\left(x_{n}-\gamma_{n} A^{*}\left(A x_{n}-B y_{n}\right)\right)> \\
& +<x_{n}-x, x_{n}-x-\gamma_{n} A^{*}\left(A x_{n}-B y_{n}\right)> \\
= & <x_{n}-\gamma_{n} A^{*}\left(A x_{n}-B y_{n}\right)-x+\gamma_{n} A^{*}\left(A x_{n}-B y_{n}\right) \\
& ,\left(T_{1}-I\right)\left(x_{n}-\gamma_{n} A^{*}\left(A x_{n}-B y_{n}\right)\right)> \\
& +\left\|x_{n}-x\right\|^{2}-<x_{n}-x, \gamma_{n} A^{*}\left(A x_{n}-B y_{n}\right)> \\
= & <x_{n}-\gamma_{n} A^{*}\left(A x_{n}-B y_{n}\right)-x,\left(T_{1}-I\right)\left(x_{n}-\gamma_{n} A^{*}\left(A x_{n}-B y_{n}\right)\right)> \\
& +<\gamma_{n} A^{*}\left(A x_{n}-B y_{n}\right),\left(T_{1}-I\right)\left(x_{n}-\gamma_{n} A^{*}\left(A x_{n}-B y_{n}\right)\right)> \\
& +\left\|x_{n}-x\right\|^{2}-\gamma_{n}<A x_{n}-A x, A x_{n}-B y_{n}> \\
\leq & -\frac{1-k}{2}\left\|\left(I-T_{1}\right)\left(x_{n}-\gamma_{n} A^{*}\left(A x_{n}-B y_{n}\right)\right)\right\|^{2} \\
& +<\gamma_{n} A^{*}\left(A x_{n}-B y_{n}\right),\left(T_{1}-I\right)\left(x_{n}-\gamma_{n} A^{*}\left(A x_{n}-B y_{n}\right)\right)> \\
& +\left\|x_{n}-x\right\|^{2}-\gamma_{n}<A x_{n}-A x, A x_{n}-B y_{n}> \\
\leq & -\frac{1-k}{2}\left\|\left(I-T_{1}\right)\left(x_{n}-\gamma_{n} A^{*}\left(A x_{n}-B y_{n}\right)\right)\right\|^{2} \\
& +\left\|\gamma_{n} A^{*}\left(A x_{n}-B y_{n}\right)\right\|\left\|\left(I-T_{1}\right)\left(x_{n}-\gamma_{n} A^{*}\left(A x_{n}-B y_{n}\right)\right)\right\| \\
& +\left\|x_{n}-x\right\|^{2}-\gamma_{n}<A x_{n}-A x, A x_{n}-B y_{n}>.
\end{aligned}
$$

On the other hand, since

$$
\begin{aligned}
\| x_{n}- & \gamma_{n} A^{*}\left(A x_{n}-B y_{n}\right)-x \|^{2} \\
& =\left\|x_{n}-x\right\|^{2}+\left\|\gamma_{n} A^{*}\left(A x_{n}-B y_{n}\right)\right\|^{2}-2 \gamma_{n}<x_{n}-x, A^{*}\left(A x_{n}-B y_{n}\right)> \\
& \left.=\left\|x_{n}-x\right\|^{2}+\left\|\gamma_{n} A^{*}\left(A x_{n}-B y_{n}\right)\right\|^{2}-2 \gamma_{n}<A x_{n}-A x, A x_{n}-B y_{n}\right)>
\end{aligned}
$$


and

$$
\begin{aligned}
\left\|\gamma_{n} A^{*}\left(A x_{n}-B y_{n}\right)\right\|^{2} & =\gamma_{n}^{2}<A^{*}\left(A x_{n}-B y_{n}\right), A^{*}\left(A x_{n}-B y_{n}\right)> \\
& =\gamma_{n}^{2}<A x_{n}-B y_{n}, A A^{*}\left(A x_{n}-B y_{n}\right)> \\
& \leq \lambda_{A} \gamma_{n}^{2}<A x_{n}-B y_{n}, A x_{n}-B y_{n}> \\
& =\lambda_{A} \gamma_{n}^{2}\left\|A x_{n}-B y_{n}\right\|^{2}
\end{aligned}
$$

Combine (3.1), (3.2), (3.3) and (3.4), then we have

$$
\begin{aligned}
&\left\|x_{n+1}-x\right\|^{2} \\
& \leq \alpha_{n}^{2}\left\|x_{n}-x\right\|^{2}+\left(1-\alpha_{n}\right)^{2}\left\{\left\|x_{n}-x\right\|^{2}-2 \gamma_{n}<A x_{n}-A x, A x_{n}-B y_{n}>\right. \\
&\left.+\left\|\gamma_{n} A^{*}\left(A x_{n}-B y_{n}\right)\right\|^{2}+k\left\|\left(I-T_{1}\right)\left(x_{n}-\gamma_{n} A^{*}\left(A x_{n}-B y_{n}\right)\right)\right\|^{2}\right\} \\
&+2 \alpha_{n}\left(1-\alpha_{n}\right)\left\{-\frac{1-k}{2}\left\|\left(I-T_{1}\right)\left(x_{n}-\gamma_{n} A^{*}\left(A x_{n}-B y_{n}\right)\right)\right\|^{2}\right. \\
&+\left\|\gamma_{n} A^{*}\left(A x_{n}-B y_{n}\right)\right\|\left\|\left(I-T_{1}\right)\left(x_{n}-\gamma_{n} A^{*}\left(A x_{n}-B y_{n}\right)\right)\right\| \\
&\left.+\left\|x_{n}-x\right\|^{2}-\gamma_{n}<A x_{n}-A x, A x_{n}-B y_{n}>\right\} \\
&=\left\{\alpha_{n}^{2}+2 \alpha_{n}\left(1-\alpha_{n}\right)+\left(1-\alpha_{n}\right)^{2}\right\}\left\|x_{n}-x\right\|^{2} \\
&+\left\{-2\left(\frac{1-k}{2}\right) \alpha_{n}\left(1-\alpha_{n}\right)+k\left(1-\alpha_{n}\right)^{2}\right\}\left\|\left(I-T_{1}\right)\left(x_{n}-\gamma_{n} A^{*}\left(A x_{n}-B y_{n}\right)\right)\right\|^{2} \\
&+2 \alpha_{n}\left(1-\alpha_{n}\right)\left\|\gamma_{n} A^{*}\left(A x_{n}-B y_{n}\right)\right\|\left\|\left(I-T_{1}\right)\left(x_{n}-\gamma_{n} A^{*}\left(A x_{n}-B y_{n}\right)\right)\right\| \\
&-\left\{2 \alpha_{n}\left(1-\alpha_{n}\right)+2\left(1-\alpha_{n}\right)^{2}\right\} \gamma_{n}<A x_{n}-A x, A x_{n}-B y_{n}> \\
&+\left(1-\alpha_{n}\right)^{2}\left\|\gamma_{n} A^{*}\left(A x_{n}-B y_{n}\right)\right\|^{2} \\
&\left\|x_{n}-x\right\|^{2}+\left(1-\alpha_{n}\right)\left(k-\alpha_{n}\right)\left\|\left(I-T_{1}\right)\left(x_{n}-\gamma_{n} A^{*}\left(A x_{n}-B y_{n}\right)\right)\right\|^{2} \\
&+\left\{\alpha_{n}^{2}\left\|\gamma_{n} A^{*}\left(A x_{n}-B y_{n}\right)\right\|^{2}+\left(1-\alpha_{n}\right)^{2}\left\|\left(I-T_{1}\right)\left(x_{n}-\gamma_{n} A^{*}\left(A x_{n}-B y_{n}\right)\right)\right\|^{2}\right\} \\
&-\left\{2 \alpha_{n}\left(1-\alpha_{n}\right)+2\left(1-\alpha_{n}\right)^{2}\right\} \gamma_{n}<A x_{n}-A x, A x_{n}-B y_{n}> \\
&+\left(1-\alpha_{n}\right)^{2}\left\|\gamma_{n} A^{*}\left(A x_{n}-B y_{n}\right)\right\|^{2} \\
&=\left\|x_{n}-x\right\|^{2}+\left\{\left(1-\alpha_{n}\right)\left(k+1-2 \alpha_{n}\right)\right\}\left\|\left(I-T_{1}\right)\left(x_{n}-\gamma_{n} A^{*}\left(A x_{n}-B y_{n}\right)\right)\right\|^{2} \\
&- 2\left(1-\alpha_{n}\right) \gamma_{n}<A x_{n}-A x, A x_{n}-B y_{n}> \\
&+\left(\left(1-\alpha_{n}\right)^{2}+\alpha_{n}^{2}\right)\left\|\gamma_{n} A^{*}\left(A x_{n}-B y_{n}\right)\right\|^{2} . \\
&
\end{aligned}
$$

Similarly, from the second equality of algorithm we can get

$$
\begin{aligned}
\left\|y_{n+1}-y\right\|^{2}= & \left\|y_{n}-x\right\|^{2}+\left\{\left(1-\alpha_{n}\right)\left(k+1-2 \alpha_{n}\right)\right\}\left\|\left(I-T_{2}\right)\left(y_{n}+\gamma_{n} B^{*}\left(A x_{n}-B y_{n}\right)\right)\right\|^{2} \\
& +2\left(1-\alpha_{n}\right) \gamma_{n}<B y_{n}-B y, A x_{n}-B y_{n}> \\
& +\left(\left(1-\alpha_{n}\right)^{2}+\alpha_{n}^{2}\right)\left\|\gamma_{n} B^{*}\left(A x_{n}-B y_{n}\right)\right\|^{2} .
\end{aligned}
$$


Since $(x, y) \in \Omega$ so we have the fact that $A x=B y$, and finally we have

$$
\begin{aligned}
\left\|x_{n+1}-x\right\|^{2}+ & \left\|y_{n+1}-y\right\|^{2} \\
\leq & \left\|x_{n}-x\right\|^{2}+\left\|y_{n}-y\right\|^{2} \\
& -\left\{\left(1-\alpha_{n}\right)\left(2 \alpha_{n}-k-1\right)\right\}\left\|\left(I-T_{1}\right)\left(x_{n}-\gamma_{n} A^{*}\left(A x_{n}-B y_{n}\right)\right)\right\|^{2} \\
& -\left\{\left(1-\alpha_{n}\right)\left(2 \alpha_{n}-k-1\right)\right\}\left\|\left(I-T_{2}\right)\left(y_{n}+\gamma_{n} B^{*}\left(A x_{n}-B y_{n}\right)\right)\right\|^{2} \\
& -\gamma_{n}\left\{2\left(1-\alpha_{n}\right)-\left(\left(1-\alpha_{n}\right)^{2}+\alpha_{n}^{2}\right) \gamma_{n}\left(\lambda_{A}+\lambda_{B}\right)\right\}\left\|A x_{n}-B y_{n}\right\|^{2} \\
\leq & \left\|x_{n}-x\right\|^{2}+\left\|y_{n}-y\right\|^{2} \\
& -\left\{\left(1-\alpha_{n}\right)\left(2 \alpha_{n}-k-1\right)\right\}\left\|\left(I-T_{1}\right)\left(x_{n}-\gamma_{n} A^{*}\left(A x_{n}-B y_{n}\right)\right)\right\|^{2} \\
& -\left\{\left(1-\alpha_{n}\right)\left(2 \alpha_{n}-k-1\right)\right\}\left\|\left(I-T_{2}\right)\left(y_{n}+\gamma_{n} B^{*}\left(A x_{n}-B y_{n}\right)\right)\right\|^{2} \\
& -\gamma_{n}\left\{2(1-\beta)-\left((1-\alpha)^{2}+\beta^{2}\right) \gamma_{n}\left(\lambda_{A}+\lambda_{B}\right)\right\}\left\|A x_{n}-B y_{n}\right\|^{2} .
\end{aligned}
$$

Let $\Omega_{n}(x, y):=\left\|x_{n}-x\right\|^{2}+\left\|y_{n}-y\right\|^{2}$ then we have

$$
\begin{aligned}
\Omega_{n+1}(x, y) \leq & \Omega_{n}(x, y)-\gamma_{n}\left\{2(1-\beta)-\left((1-\alpha)^{2}+\beta^{2}\right) \gamma_{n}\left(\lambda_{A}+\lambda_{B}\right)\left\|A x_{n}-B y_{n}\right\|^{2}\right. \\
& -\left\{\left(1-\alpha_{n}\right)\left(2 \alpha_{n}-k-1\right)\right\}\left\|\left(I-T_{1}\right)\left(x_{n}-\gamma_{n} A^{*}\left(A x_{n}-B y_{n}\right)\right)\right\|^{2} \\
& -\left\{\left(1-\alpha_{n}\right)\left(2 \alpha_{n}-k-1\right)\right\}\left\|\left(I-T_{2}\right)\left(y_{n}+\gamma_{n} B^{*}\left(A x_{n}-B y_{n}\right)\right)\right\|^{2} .
\end{aligned}
$$

Obviously the sequence $\left\{\Omega_{n}(x, y)\right\}$ is decreasing and has lower bounded, so it converges to some finite limit $\omega(x, y)$. This means that the first condition of Lemma 2.4 (Opial's lemma) is satisfied with $W=\Omega, \mu_{n}:=\left(x_{n}, y_{n}\right), \mu^{*}:=(x, y)$. And by passing to limit in (3.8), we obtain that

$$
\lim _{n \rightarrow \infty}\left\|A x_{n}-B y_{n}\right\|=0
$$

and

$$
\begin{aligned}
& \lim _{n \rightarrow \infty}\left\|\left(I-T_{1}\right)\left(x_{n}-\gamma_{n} A^{*}\left(A x_{n}-B y_{n}\right)\right)\right\|=0, \\
& \lim _{n \rightarrow \infty}\left\|\left(I-T_{2}\right)\left(y_{n}+\gamma_{n} B^{*}\left(A x_{n}-B y_{n}\right)\right)\right\|=0 .
\end{aligned}
$$

Since $\left\|x_{n}-x\right\| \leq \Omega_{n}(x, y),\left\|y_{n}-y\right\| \leq \Omega_{n}(x, y)$ and $\lim _{n \rightarrow \infty} \Omega_{n}(x, y)$ exists, we know that $\left\{x_{n}\right\}$ and $\left\{y_{n}\right\}$ are bounded, and $\lim _{\sup _{n \rightarrow \infty}}\left\|x_{n}-x\right\|$ and $\lim _{\sup _{n \rightarrow \infty}} \| y_{n}-$ $y \|$ exist. Let $x^{*}$ and $y^{*}$ be respectively weak cluster points of the sequences $\left\{x_{n}\right\}$ and $\left\{y_{n}\right\}$, Further, $\left\{x_{n}-\gamma_{n} A^{*}\left(A x_{n}-B y_{n}\right)\right\}$ also converges weakly to $x^{*},\left\{y_{n}+\right.$ $\left.\gamma_{n} B^{*}\left(A x_{n}-B y_{n}\right)\right\}$ converges weakly to $y^{*}$. From Lemma 2.5 , we have

$$
\begin{aligned}
\| x_{n+1} & -x_{n}\left\|^{2}=\right\| x_{n+1}-x-x_{n}+x \|^{2} \\
& =\left\|x_{n+1}-x\right\|^{2}-\left\|x_{n}-x\right\|^{2}-2<x_{n+1}-x_{n}, x_{n}-x> \\
& =\left\|x_{n+1}-x\right\|^{2}-\left\|x_{n}-x\right\|^{2}-2<x_{n+1}-x^{*}, x_{n}-x>+2<x_{n}-x^{*}, x_{n}-x>,
\end{aligned}
$$


SO

$$
\limsup _{n \rightarrow \infty}\left\|x_{n+1}-x_{n}\right\|=0
$$

Similarly

$$
\limsup _{n \rightarrow \infty}\left\|y_{n+1}-y_{n}\right\|=0
$$

Since k-strictly pseudocontractive mapping Lipschitz continuous with a constant $\frac{1+k}{1-k}$, from Lemma 2.3, we obtain

$$
\begin{aligned}
\left\|x_{n}-T_{1} x_{n}\right\|= & \left\|x_{n}-x_{n+1}+x_{n+1}-T_{1} x_{n}\right\| \\
\leq & \left\|x_{n}-x_{n+1}\right\|+\left\|x_{n+1}-T_{1} x_{n}\right\| \\
= & \left\|x_{n}-x_{n+1}\right\|+\left\|\alpha_{n} x_{n}+\left(1-\alpha_{n}\right) T_{1}\left(x_{n}-\gamma_{n} A^{*}\left(A x_{n}-B y_{n}\right)\right)-T_{1} x_{n}\right\| \\
\leq & \left\|x_{n}-x_{n+1}\right\|+\alpha_{n}\left\|x_{n}-T_{1} x_{n}\right\| \\
& +\left(1-\alpha_{n}\right)\left\|T_{1}\left(x_{n}-\gamma_{n} A^{*}\left(A x_{n}-B y_{n}\right)\right)-T_{1} x_{n}\right\| \\
\leq & \left.\left\|x_{n}-x_{n+1}\right\|+\alpha_{n}\left\|x_{n}-T_{1} x_{n}\right\|+\left(1-\alpha_{n}\right) \frac{1+k}{1-k} \|-\gamma_{n} A^{*}\left(A x_{n}-B y_{n}\right)\right) \| .
\end{aligned}
$$

It follows from (3.9) and (3.13) that

$$
\lim _{n \rightarrow \infty}\left\|x_{n}-T_{1} x_{n}\right\|=0
$$

Similarly

$$
\lim _{n \rightarrow \infty}\left\|y_{n}-T_{2} y_{n}\right\|=0
$$

Since $\left\{x_{n}\right\}$ and $\left\{y_{n}\right\}$ converges weakly to $x^{*}$ and $y^{*}$, respectively, then it follows from (3.10), (3.11), (3.16), (3.17) and Lemma 2.3 that $x^{*} \in F\left(T_{1}\right)$ and $y^{*} \in F\left(T_{2}\right)$.

On the other hand, since the squared norm is weakly lower semicontinuous, we have

$$
\left\|A x^{*}-B y^{*}\right\|^{2} \leq \operatorname{limin} f_{n \rightarrow \infty}\left\|A x_{n}-B_{n}\right\|^{2}=0,
$$

therefore $A x^{*}=B y^{*}$. This implies that $\left(x^{*}, y^{*}\right) \in \Omega$. Thus from Lemma 2.4, we know that $\left\{\left(x_{n}, y_{n}\right)\right\}$ converges weakly to $\left(x^{*}, y^{*}\right)$. The proof of conclusion(I) is completed.

Next, we prove the conclusion(II).

Since $T_{1}$ and $T_{2}$ are semi-compact, $\left\{x_{n}\right\}$ and $\left\{y_{n}\right\}$ are bounded and $\lim _{n \rightarrow \infty} \| x_{n}-$ $T_{1} x_{n}\left\|=0, \lim _{n \rightarrow \infty}\right\| y_{n}-T_{2} y_{n} \|=0$, then there exist subsequences $\left\{x_{n_{j}}\right\}$ and $\left\{y_{n_{j}}\right\}$ of $\left\{x_{n}\right\}$ and $\left\{y_{n}\right\}$ such that $\left\{x_{n_{j}}\right\}$ and $\left\{y_{n_{j}}\right\}$ converge strongly to $x^{*}$ and $y^{*}$, respectively. From Lemma 2.3, we have $x^{*} \in F\left(T_{1}\right)$ and $y^{*} \in F\left(T_{2}\right)$. Further, since the squared norm is weakly lower semicontinuous and $A x_{n_{j}}-B y_{n_{j}} \rightarrow A x^{*}-B y^{*}$, we have

$$
\left\|A x^{*}-B y^{*}\right\|^{2} \leq \liminf _{j \rightarrow \infty}\left\|A x_{n_{j}}-B_{n_{j}}\right\|^{2}=0
$$


so $A x^{*}=B y^{*}$. This implies that $\left(x^{*}, y^{*}\right) \in \Omega$.

On the other hand, since $\Omega_{n}(x, y)=\left\|x_{n}-x\right\|^{2}+\left\|y_{n}-y\right\|^{2}$ for any $(x, y) \in \Omega$, we know that $\lim _{j \rightarrow \infty} \Omega_{n_{j}}\left(x^{*}, y^{*}\right)=0$. From conclusion(I), we have $\lim _{n \rightarrow \infty} \Omega_{n}\left(x^{*}, y^{*}\right)$ exists, therefore $\lim _{n \rightarrow \infty} \Omega_{n}\left(x^{*}, y^{*}\right)=0$. Further, we can obtain that $\lim _{n \rightarrow \infty} \| x_{n}-$ $x^{*} \|=0$ and $\lim _{n \rightarrow \infty}\left\|y_{n}-y^{*}\right\|=0$. This completes the proof of the conclusion(II).

For nonexpansive mapping, we have $k=0$ in Theorem 3.1 to obtain the following corollary.

Corollary 3.2. Let $H_{1}, H_{2}, H_{3}$ be real Hilbert spaces, let $T_{1}: H_{1} \rightarrow H_{1}$, $T_{2}: H_{2} \rightarrow H_{2}$ be two nonexpansive mappings, and $A: H_{1} \rightarrow H_{3}, B: H_{2} \rightarrow H_{3}$ be two bounded linear operators. Assume that the iteration scheme $\left\{\left(x_{n}, y_{n}\right)\right\}$ is defined as follows:

$$
\left\{\begin{array}{l}
\forall x_{1} \in H_{1}, \quad \forall y_{1} \in H_{2} ; \\
x_{n+1}=\alpha_{n} x_{n}+\left(1-\alpha_{n}\right) T_{1}\left(x_{n}-\gamma_{n} A^{*}\left(A x_{n}-B y_{n}\right)\right) ; \\
y_{n+1}=\alpha_{n} y_{n}+\left(1-\alpha_{n}\right) T_{2}\left(y_{n}+\gamma_{n} B^{*}\left(A x_{n}-B y_{n}\right)\right), \quad \forall n \geq 1 .
\end{array}\right.
$$

where $\lambda_{A}$ and $\lambda_{B}$ stand for the spectral radius of $A^{*} A$ and $B^{*} B$ respectively, $\left\{\alpha_{n}\right\}$ is a sequence in $(0,1)$ with $\frac{1}{2}<\alpha \leq \alpha_{n} \leq \beta<1$ (for some $\alpha, \beta \in(0,1)$ ), and $\left\{\gamma_{n}\right\}$ is a positive real sequence such that $\gamma_{n} \in\left(\varepsilon, \frac{2(1-\beta)}{\left((1-\alpha)^{2}+\beta^{2}\right)\left(\lambda_{A}+\lambda_{B}\right)}-\varepsilon\right)$ (for $\varepsilon$ small enough), $C:=F\left(T_{1}\right)$ and $Q:=F\left(T_{2}\right)$. If $\Omega \neq \emptyset$, then

(I) The sequence $\left\{\left(x_{n}, y_{n}\right)\right\}$ converges weakly to a solution $(x, y)$ of the problem (1.4).

(II) In addition, if $T_{1}, T_{2}$ are also semi-compact, then $\left\{\left(x_{n}, y_{n}\right)\right\}$ converges strongly to a solution of the problem (1.4).

Remark 3.3. The iterative method of Theorem 3.1 reduces to the iterative method in [12] when $\alpha_{n}=0$ for all $n \geq 1$. The results obtained in this paper extend the results from firmly quasi-nonexpansive mappings and nonexpansive mappings to more general strictly pseudocontractive mappings. So, our results improve and extend the results of Moudafi $[11,12]$.

\section{References}

[1] C. Byne, Iterative obligue projection onto convex sets and the split feasibility problem, Inverse Problems, Vol. 18(2002) 441-453. http://dx.doi.org/10.1088/0266-5611/18/2/310

[2] Y. Censor and T. Elfving, A multiprojection algorithm using Bregman projections in a product space, Numerical Algorithms, Vol. 8(1994), 221-239. http://dx.doi.org/10.1007/bf02142692 
[3] Y. Censor, T. Bortfeld, B.Martin, A. Trofimov, A unified approach for inverse problem in intensity-modulated radiation therapy, phys. Med. Biol. 51(2006), 2352-2365. http://dx.doi.org/10.1088/0031-9155/51/10/001

[4] Y. Censor, T.Elfving, N. Kopf, T. Bortfeld, The multiple-sets split feasibility problem and its applications, Inverse Problem 21(2005), 2071-2084. http://dx.doi.org/10.1088/0266-5611/21/6/017

[5] Y. Censor, A. Motova, A. Segal, Pertured projections and subgradient projections for the multiple-sets split feasibility problem, J. Math. Anal. Appl. 327(2007), 1244-1256. http://dx.doi.org/10.1016/j.jmaa.2006.05.010

[6] P.L. Combettes, Hilbertian convex feasibility problem: Convergence of projection methods, Applied Mathematics and Optimization, 35(1997), 311-330. http://dx.doi.org/10.1007/s002459900050

[7] B. Qu, N. Xiu, A note on the CQ algorithm for the split feasibility problem, Inverse Probl. 21(2005), 1655-1665. http://dx.doi.org/10.1088/0266$5611 / 21 / 5 / 009$

[8] A. Aleyner, S. Reich, Block-iterative algorithms for solving convex feasibility problems in Hilbert and in Banach, Journal of Mathematical Analysis and Applications, 343(2008), 427-435. http://dx.doi.org/10.1016/j.jmaa.2008.01.087

[9] Q. Yang, The relaxed CQ algorithm solving the split feasibility problem, Inverse Problem 20(2004), 103-120.

[10] H. K. Xu, Iterative methods for split feasibility problem in infinitedimensional Hilbert spaces, Inverse Problem, 26(2010), 105018(17pp). http://dx.doi.org/10.1088/0266-5611/26/10/105018

[11] A. Moudafi, A relaxed alternating CQ-algorithm for convex feasibility problems, Nonlinear Analysis 79(2013), 117-121. http://dx.doi.org/10.1016/j.na.2012.11.013

[12] A. Moudafi, Eman Al-Shemas, Simultaneous iterative methods for split equality problems, Mathematical Programming and Applications Volume 1(2013), No. $2,1-11$.

[13] Acedo, GL, Xu, HK: Iterative methods for strict pseudocontractions in Hilbert spaces. Nonlinear Anal. 67, 2258-2271 (2007) http://dx.doi.org/10.1016/j.na.2006.08.036 
[14] G. Marino. H.K. Xu, Weak and strong convergence theorms for strict pseudocontractions in Hilbert space, J. Math. Anal. Appl. 329(2007) 336-346. http://dx.doi.org/10.1016/j.jmaa.2006.06.055

Received: October 17, 2014; Published: November 24, 2014 